PAPER

\title{
Prognosis of asymptomatic stenosis of the middle cerebral artery
}

\author{
C Kremer, T Schaettin, D Georgiadis, R W Baumgartner
}

J Neurol Neurosurg Psychiatry 2004;75:1300-1303. doi: 10.1136/jnnp.2003.017863

See end of article for authors' affiliations

.....................

Correspondence to: Dr Christine Kremer,

Neurosurgery Department,

University Heidelberg,

Faculty of Clinical

Medicine Mannheim

Theodor-Kutzer-Ufer 1-3,

D-68135 Mannheim,

Germany;

christine.kremer@nch.ma.

uni-heidelberg.de

Received 2 May 2003

Revised 17November 2003

Accepted

21 December 2003

\begin{abstract}
Background: The risk of ischaemic events in asymptomatic intracranial atherosclerosis is unknown.
Objective: To follow up patients with asymptomatic atherosclerotic middle cerebral artery stenosis (MCAS) to evaluate the long term stroke risk in its territory.

Methods: Consecutive white patients with asymptomatic atherosclerotic MCAS were enrolled. Patients with MCAS of possible or proven non-atherosclerotic origin were excluded. MCAS was assessed by transcranial colour duplex sonography according to published angiography validated criteria. Medical treatment was given at the discretion of the treating physician.

Results: 50 patients were included and followed for (mean (SD)) 815 (351) days; three were lost to follow up. MCAS was $<50 \%$ in 38 and $\geqslant 50 \%$ in 12 . No patient suffered an ischaemic event in the MCAS territory; one had a transient ischaemic attack in the contralateral hemisphere. Three patients died, one from a subdural haematoma in the contralateral hemisphere, and two from non-stroke-related causes. Medical treatment at baseline included antithrombotic drugs in 42 cases (antiplatelet agent, $n=39$; warfarin, $n=3$ ), and statins in 22; at the end of follow up 45 of the 47 survivors were on antithrombotic drugs (antiplatelet agent, $n=37$; warfarin, $n=8$ ), and 30 were on statins.

Conclusions: Asymptomatic MCAS of atherosclerotic origin appears to have a benign long term prognosis with a low risk of ipsilateral stroke in medically treated white patients.
\end{abstract}

A therosclerotic intracranial stenoses are responsible for ischaemic stroke in $5-10 \%$ of white patients, and in up to $33 \%$ of Asian patients. ${ }^{1-3}$ Patients with symptomatic intracranial atherosclerosis who suffer subsequent ischaemic events and fail to take antithrombotic treatment have very high rates of recurrent stroke or death. ${ }^{4}$ Corresponding data on the long term follow up in patients with asymptomatic intracranial atherosclerosis are lacking.

This observational study was undertaken to assess the risk of an ischaemic stroke in patients with asymptomatic stenosis of the middle cerebral artery (MCAS).

\section{METHODS}

Between July 1997 and December 2000, 2675 patients were examined using transcranial colour duplex sonography (TCCS) in the division of neuroangiology of the department of neurology of the University Hospital of Zürich. Their clinical and examination data were collected prospectively in the departmental database. ${ }^{5}$ These data were retrospectively screened for patients with asymptomatic MCAS.

Sixty five white patients were identified; 45 of these had no acute symptoms of cerebral ischaemia, while 20 were examined during a diagnostic work up for first ever ischaemic stroke. All patients were invited to a clinical follow up visit; those who declined the follow up visit had a standardised telephone interview.

The principal inclusion criterion for the study was the presence of asymptomatic MCAS that was assumed to be of atherosclerotic origin. MCAS was considered asymptomatic if no ischaemic event had occurred in the territory supplied by the stenosed middle cerebral artery before inclusion in the study. A diagnosis of MCAS was established by angiography validated TCCS criteria reported previously. ${ }^{6}$ MCAS are caused mainly by embolism from arterial, aortic, or cardiac sources, and by atherosclerosis. ${ }^{7-9}$ To avoid including patients with MCAS caused by emboli, we excluded those with the following:
- $\geqslant 50 \%$ atherosclerotic stenosis or occlusion of the ipsilateral common carotid artery or internal carotid artery;

- a known cardiac source of embolism (atrial fibrillation, sick sinus syndrome, rheumatic mitral stenosis, prosthetic heart valves, cardiac intraluminal thrombus or tumour, cardiomyopathy, recent myocardial infarct occurring less than three months earlier, left ventricular aneurysm or akinesia after myocardial infarct, endocarditis, patent foramen ovale with acute deep venous thrombosis, pulmonary embolism or atrial septum aneurysm, and paradoxical embolism).

Further exclusions were as follows:

- suspicion or presence of another non-atherosclerotic cause of MCAS suggested by intracranial dissection or fibromuscular dysplasia, Moya-Moya disease, or vasculitis (Takayasu disease, giant cell arteritis, collagen vascular disease, systemic necrotising vasculitis, granulomatous angiitis of the nervous system);

- surgical or endovascular treatment of the ipsilateral internal carotid artery;

- cerebral vascular malformation or arteriovenous fistula;

- uncontrolled hypertension (systolic pressure > $185 \mathrm{~mm} \mathrm{Hg}$ and/or diastolic pressure $>110 \mathrm{~mm} \mathrm{Hg}$ );

- severe illness (active cancer or significant liver or renal disease) or disability; alcohol, or illicit drug abuse.

\section{Baseline investigations}

The following risk factors for ischaemic stroke were assessed: current cigarette smoking (cigarette smoking within the last five years); former cigarette smoking (abstention from cigarette smoking for more than five years); hypertension,

Abbreviations: MCAS, middle cerebral artery stenosis; TCCS, transcranial colour duplex sonography 
defined by preadmission history and medical records; diabetes mellitus, defined as a fasting venous plasma glucose concentration of $\geqslant 7.8 \mathrm{mmol} / \mathrm{l}$ on at least two separate occasions, and/or $\geqslant 11.1 \mathrm{mmol} / \mathrm{l}$ two hours after the ingestion of $75 \mathrm{~g}$ of glucose and on one other occasion during the two hour test; hypercholesterolaemia, defined as a total venous plasma cholesterol level above $5 \mathrm{mmol} / \mathrm{l}$; history of coronary heart disease and peripheral artery disease; family history of stroke; and history of myocardial infarction.

Ischaemic deficits were classified according to their duration and location as stroke $(>24$ hours), transient ischaemic attack (TIA; $\leqslant 24$ hours), retinal infarct $(>24$ hours), and amaurosis fugax $(\leqslant 24$ hours). Patients with ischaemic deficits also had cranial computed tomography (CT), magnetic resonance imaging (MRI), or both.

\section{Ultrasound investigations}

Ultrasound studies were done using the same equipment throughout (for extracranial and transcranial colour duplex studies: Acuson XP 10 or Sequoia, Mountain View, California, USA) according to diagnostic criteria reported recently, and are summarised below. ${ }^{5}$

\section{TCCS studies}

TCCS studies of the basal cerebral arteries were carried out using sector transducers (2.0 to $3.5 \mathrm{MHz}$ ). The transtemporal approach was used for insonation of the terminal (C1) internal carotid artery, middle cerebral artery, anterior cerebral artery, precommunicating (Pl) and postcommunicating (P2) posterior cerebral arteries, and anterior and posterior communicating arteries. Transforaminal (transnuchal) insonation was done to assess the atlas loop (V3) and the intracranial segment (V4) of both vertebral and basilar arteries. Intracranial arteries were investigated for the presence of stenoses, occlusions, and cross flow through the anterior and posterior communicating arteries according to previously published criteria. ${ }^{6}{ }^{10}{ }^{11}$ Briefly, flow direction and peak systolic and end diastolic velocities were documented for every insonated artery. Angle correction was located within a straight vessel segment of $>20 \mathrm{~mm}$ length. MCAS was assumed to be present with peak systolic velocity values of $>155 \mathrm{~cm} / \mathrm{s} \quad(<50 \%$ stenosis $)$ or $>220 \mathrm{~cm} / \mathrm{s} \quad(\geqslant 50 \%$ stenosis). ${ }^{6}$ Only stenoses of the Ml segment of the middle cerebral artery were assessed. Four patients with insufficient temporal bony windows were also investigated with the echocontrast agent Levovist ${ }^{\circledR}$, using concentrations of 400 $\mathrm{mg} / \mathrm{ml}^{12}$

Extracranial colour duplex sonography of the common carotid artery, internal carotid artery, external carotid artery, subclavian artery, and extracranial vertebral artery was done with linear transducers $(5-8 \mathrm{MHz})$, and of the cervical segment of the internal carotid artery with sector transducers (2.0-3.5 MHz). Stenoses and occlusions were quantified according to previously published criteria. ${ }^{13} 14$

Baseline characteristics and ultrasound findings are shown in tables 1 and 2, respectively. MCAS was equally frequent in both sexes, and hypertension was the most common risk factor. Of 13 patients with first ever ischaemic stroke, 11 presented with strokes in the anterior circulation contralateral to the asymptomatic MCAS and two with posterior circulation stroke.

\section{Antithrombotic and lipid lowering treatment at inclusion and follow up}

These results are shown in table 3. Forty two patients (84\%) received antithrombotic agents at baseline (aspirin, $\mathrm{n}=36$; aspirin plus dipyridamole, $\mathrm{n}=3$; warfarin, $\mathrm{n}=3$ ). The indication for antiplatelet therapy was stroke prevention in 27 patients who had suffered cerebral ischaemic events, and
Table 1 Baseline characteristics in 50 patients with asymptomatic middle cerebral artery stenoses

\begin{tabular}{ll}
\hline Men/women & $25(50 \%) / 25(50 \%)$ \\
Age (years) (mean (SD)) & $67(11)$ \\
Cigarette smoking & $31(62 \%)$ \\
Present/former smoker & $17(56 \%) / 14(45 \%)$ \\
Hypertension & $38(75 \%)$ \\
Diabetes mellitus & $9(18 \%)$ \\
Hypercholesterolaemia & $26(52 \%)$ \\
Coronary artery disease & $17(34 \%)$ \\
Peripheral artery disease & $15(30 \%)$ \\
History of myocardial infarction & $8(16 \%)$ \\
Ischaemic stroke & $13(26 \%)$ \\
Contralateral hemisphere, MCA territory & $9(18 \%)$ \\
ACA territory & $2(4 \%)$ \\
Vertebrobasilar territory & $2(4 \%)$ \\
Subdural haematoma in contralateral & $1(2 \%)$ \\
hemisphere & $11(22 \%)$ \\
Transient ischaemic attack & $3(6 \%)$ \\
Amaurosis fugax & $1(2 \%)$ \\
Retinal infarct &
\end{tabular}

Values are $\mathrm{n}(\%)$ unless indicated.

ACA anterior cerebral artery; MCA, middle cerebral artery

Contralateral, hemisphere opposite to the stenosed MCA

cardiovascular disease in 12 patients (coronary artery disease, $\mathrm{n}=9$; coronary artery disease and peripheral artery disease, $\mathrm{n}=3$ ). Warfarin was given in two cases because of coronary artery disease, and for stroke prevention in a patient with a symptomatic and severe stenosis of the opposite internal carotid artery.

At the end of follow up, 45 of 47 survivors (96\%) had received antithrombotic therapy (aspirin, $\mathrm{n}=31$; aspirin plus dipyridamole, $\mathrm{n}=2$; clopidogrel, $\mathrm{n}=4$; warfarin, $\mathrm{n}=8$ ). During follow up, warfarin was started in two patients with coronary artery disease who developed new angina; in four patients aspirin was replaced by warfarin because of the development of coronary artery disease with angina in two cases, recurrent TIAs in one case, and the development of peripheral artery disease in the remaining case. Lipid lowering drugs included only statins, which were given at baseline in 22 of 50 patients (44\%), and at follow up in 30 of 47 survivors $(64 \%)$. The finding of asymptomatic MCAS did not alter treatment in any case.

\section{RESULTS}

Fifty three patients with a mean (SD) age of 67 (11) years (range 30 to 84) fulfilled the inclusion criteria, and 50 (25 men, 25 women) could be followed up for a mean period of 815 (351) days (range 314 to 1642); three patients were lost to follow up and were excluded from the study. Twelve further patients with MCAS were identified from the database, but were excluded because of the presence of one or more exclusion criteria (atrial fibrillation or other cardiac embolic source, $\mathrm{n}=5$; ipsilateral internal carotid artery of common carotid artery stenosis of $>50 \%, \mathrm{n}=6$; myocardial infarction less than three months previously, $\mathrm{n}=1$ ). Thirty seven patients had a clinical follow up including TCCS, 10 had telephone interviews, and three died during follow up. The degree of MCAS was $<50 \%$ in 38 cases $(76 \%)$ and $\geqslant 50 \%$ in 12 cases $(24 \%)$.

\section{Indications for ultrasound investigations}

Twenty nine patients had ultrasound examinations as part of the diagnostic work up of a stroke $(n=13)$, TIA $(n=11)$, amaurosis fugax $(n=3)$, or retinal infarct $(n=1)$, which occurred before inclusion in the study (table 1). One patient had a subdural haematoma in the contralateral hemisphere. The indications for the ultrasound investigations of the remaining 21 patients without cerebral ischaemia before 
Table 2 Extracranial and additional intracranial cerebral artery stenoses and occlusions in 50 patients with asymptomatic middle cerebral artery stenoses

\begin{tabular}{|c|c|c|c|}
\hline \multirow[b]{2}{*}{ Narrowed or occluded cerebral artery } & \multicolumn{2}{|l|}{ Stenosis } & \multirow[b]{2}{*}{ Occlusion } \\
\hline & $<50 \%$ & $\geqslant 50 \%$ & \\
\hline CCA ipsilateral to stenosed MCA & $30(60 \%)$ & 0 & 0 \\
\hline Contralateral & $24(48 \%)$ & $1(2 \%)$ & 0 \\
\hline ICA origin ipsilateral to stenosed MCA & $26(52 \%)$ & 0 & 0 \\
\hline Contralateral & $30(60 \%)$ & $8(16 \%)$ & $1(2 \%)$ \\
\hline VA origin ipsilateral to stenosed MCA & $1(2 \%)$ & $1(2 \%)$ & 0 \\
\hline Contralateral & $3(6 \%)$ & 0 & $1(2 \%)$ \\
\hline ACA ipsilateral to stenosed MCA & $6(12 \%)$ & $7(14 \%)$ & 0 \\
\hline Contralateral & $7(14 \%)$ & $3(6 \%)$ & 0 \\
\hline MCA contralateral to stenosed MCA & $7(14 \%)$ & $5(10 \%)$ & 0 \\
\hline PCA ipsilateral to stenosed MCA & $5(10 \%)$ & $3(6 \%)$ & 0 \\
\hline Contralateral & $3(6 \%)$ & $3(6 \%)$ & 0 \\
\hline BA & $2(4 \%)$ & 0 & 0 \\
\hline
\end{tabular}

inclusion were planned cardiovascular interventions $(n=4$; coronary bypass surgery in two cases, and femoral bypass surgery and surgery for an aneurysm of the abdominal aorta in one case each), vertigo $(n=4)$, recurrent syncope or fainting $(n=3)$, perioperative ultrasound in patients who underwent carotid endarterectomy $(\mathrm{n}=4)$, unclear disturbance of vision $(n=2)$, search for carotid stenosis in the presence of peripheral artery disease $(n=2)$, and epileptic seizures and carotid bruit in one case each.

\section{Clinical outcome}

No patient suffered an ischaemic stroke or TIA in the territory supplied by the asymptomatic MCAS during the study period. One patient treated with aspirin $100 \mathrm{mg} / \mathrm{d}$ suffered a recurrent TIA because of a symptomatic contralateral MCAS after 1106 days.

One patient who had warfarin died from a subdural haematoma in the hemisphere opposite to the MCAS after 758 days. Two other patients died after 370 and 869 days, respectively. The cause of death was a ruptured infrarenal aortic aneurysm in one, and septic multiple organ failure after pneumonia in the other. Three additional patients developed coronary artery disease with stable angina.

\section{DISCUSSION}

The results of our study suggest that medically treated white patients with asymptomatic MCAS have a low stroke risk in the territory supplied by the narrowed vessel. To our knowledge, this is the first study evaluating the stroke risk in white subjects with asymptomatic atherosclerotic MCAS.

One possible explanation for the low stroke risk might be the fact that MCA plaque is usually fibrocalcific and thus does not represent an embolic focus, in contrast to extracranial cerebral artery stenoses. ${ }^{15}$ Numerous transcranial Doppler studies using microembolic signal detection in patients with asymptomatic MCAS are in line with this assumption, because no microembolic signals were detected in those studies, suggesting the presence of stable plaques. $^{716-18}$ The low stroke risk found in the present study contrasts with the results obtained in studies investigating patients with symptomatic MCAS. ${ }^{19-21}$ In the extracranialintracranial bypass trial, medically treated patients with symptomatic MCAS showed a $7.8 \%$ annual rate of ipsilateral stroke. ${ }^{19} 20$ In a TCCS study, 40 patients with symptomatic MCAS were followed for 26 months. An ischaemic stroke occurred in the territory of MCAS in eight cases $(20 \%)$, which corresponds to an annual stroke rate of $2.2 \%$, while six patients showed progression of their MCAS. ${ }^{21}$ In another study using microembolic signal detection by transcranial Doppler of 20 patients with MCAS, 23 arteries could be monitored. Microembolic signals were observed in five of 14 symptomatic cases of MCAS in the poststenotic segment, and in none of the nine cases of asymptomatic MCAS. ${ }^{7}$ These studies indicate that symptomatic atherosclerotic MCAS may also be unstable in the long term, in contrast to our present observations in patients with asymptomatic MCAS.

Table 3 Antithrombotic and lipid lowering treatment in 50 patients with asymptomatic middle cerebral artery stenoses at baseline and follow up

\begin{tabular}{lll}
\hline & Baseline $(\mathbf{n = 5 0 )}$ & Follow up $(\mathbf{n = 4 7})^{*}$ \\
\hline Aspirin (100-300 mg/d) & $36(72 \%)$ & $31(66 \%)$ \\
Change to clopidogrel $(75 \mathrm{mg} / \mathrm{d})$ & $1(2 \%)$ & 0 \\
Change to warfarin & $3(6 \%)$ & 0 \\
Aspirin $(50 \mathrm{mg} / \mathrm{d})+$ dipyridamole $(400 \mathrm{mg} / \mathrm{d})$ & $3(6 \%)$ & $2(4 \%)$ \\
Change to clopidogrel $(75 \mathrm{mg} / \mathrm{d})$ & $1(2 \%)$ & 0 \\
Clopidogrel $(75 \mathrm{mg} / \mathrm{d})$ & 0 & $4(9 \%)$ \\
Warfarin & $3(6 \%)$ & $8(17 \%)$ \\
$\quad$ Change to other antithrombotic therapy & 0 & 0 \\
No antithrombotic therapy & $8(16 \%)$ & $2(4 \%)$ \\
Change to aspirin (100 mg/d) & $1(2 \%)$ & 0 \\
Change to clopidogrel $(75 \mathrm{mg} / \mathrm{d})$ & $2(4 \%)$ & 0 \\
Change to warfarin & $2(4 \%)$ & 0 \\
Lipid lowering therapy (statin) & $22(44 \%)$ & $30(64 \%)$ \\
\hline Values are $\mathrm{n}(\%)$. & & \\
*Three patients died during follow up. & & \\
\hline
\end{tabular}


Half of our patients with MCAS were women, whereas two other studies had a somewhat lower percentage of women, ${ }^{20} 21$ and a third a higher percentage. ${ }^{8}$. Arterial hypertension was the main vascular risk factor in this study (75\% of cases), which is similar to the incidence of $48-75 \%$ reported in patients with symptomatic atherosclerotic MCAS. ${ }^{80-22}$

Atherosclerotic intracranial stenoses were more often the cause of ischaemic stroke in Asian than in white patients. ${ }^{1-3}$ Thus, asymptomatic MCAS could have a worse prognosis in Asian patients than we observed in the present series of white cases.

Obviously, a definitive diagnosis of atherosclerotic MCAS can only be made at necropsy. However, we presume that the MCAS identified in this study were of atherosclerotic origin because they were asymptomatic, and MCAS of embolic origin should result in acute symptoms of cerebral ischaemia. In addition, chest $x$ ray, ECG, clinical history, and clinical examination did not suggest cardiac disease. This excludes a potential cardioembolic source in the vast majority of cases. Reactive hyperaemia or collateral flow following acute stroke present an alternative diagnosis for flow acceleration in the middle cerebral artery. We must point out, though, that while 13 patients enrolled in the present study had suffered an acute ischaemic event, none of these occurred ipsilateral to the diagnosed MCAS. Collateral flow or hyperaemia would hardly be expected to cause a flow acceleration in the contralateral middle cerebral artery.

A major limitation of the present study is the small number of patients examined. This reflects the low prevalence of MCAS in the white ethnic groups. We enrolled all patients diagnosed with MCAS from among 2688 patients examined during a three year period at the University Hospital of Zürich with a catchment area of 1.2 million.

Finally, we could not study the natural course of MCAS, as $84-96 \%$ of the patients were receiving antithrombotic agents and $44-66 \%$ were receiving statins for concomitant diseases. Thus we cannot exclude the possibility that the natural course might be less benign than suggested by the present data. However, considering the fact that a randomised trial would be very difficult to conduct owing to the low prevalence of this disease, and that withdrawal of drugs for the purposes of a study is not feasible, our results represent an important estimate of the stroke risk with this vascular pathology.

\section{Conclusions}

Our data indicate that asymptomatic atherosclerotic MCAS is a benign incidental finding with a low long term risk of ipsilateral stroke in white patients treated with antithrombotic agents and statins.

\section{Authors' affiliations}

C Kremer, T Schaettin, D Georgiadis, R W Baumgartner, Department of Neurology, University Hospital of Zürich, Switzerland

Competing interests : none declared

\section{REFERENCES}

1 Sacco R, Kargman D, Gu Q, et al. Race-ethnicity and determinants of intracranial atherosclerotic cerebral infarction. The Northern Manhattan Stroke Study. Stroke 1995;26:14-20.

2 Wityk R, Lehman $D, K \operatorname{lag} M$, et al. Race and sex differences in the distribution of cerebral atherosclerosis. Stroke 1996;27:1974-80.

3 Wong K, Huang Y, Gao S, et al. Intracranial stenosis in Chinese patients with acute stroke. Neurology 1998;50:812-13.

4 Thijs V, Albers G. Symptomatic intracranial atherosclerosis. Outcome of patients who fail antithrombotic therapy. Neurology 2000:55:490-7.

5 Baumgartner RW, Sidler C, Mosso M, et al. Ischemic lacunar stroke in patients with and without other potential mechanism than small artery disease. Stroke 2003;34:653-9.

6 Baumgarmer RW, Mattle HP, Schroth G. Assessment of $\geqslant 50$ and $<50 \%$ intracranial stenoses by transcranial color-coded duplex sonography. Stroke 1999;30:87-92.

7 Segura T, Serena J, Castellanos $M$, et al. Embolism in acute middle cerebral artery stenosis. Neurology 2001;56:497-501.

8 Caplan L, Babikian C, Helgason C, et al. Occlusive disease of the middle cerebral artery. Neurology 1985;35:975-82.

9 Lhermitte F, Gautier J, Derousne C, et al. Ischemic accidents in the middle cerebral artery. A study of causes in 122 cases. Arch Neurol 1968; 19:248-56

10 Baumgartner RW, Baumgartner I, Mattle HP, et al. Transcranial color-coded duplex sonography in the evaluation of collateral flow through the circle of Willis. Am J Neuroradiol 1997;18:127-33.

11 Martin PJ, Smith JL, Gaunt ME, et al. Assessment of intracranial primary collaterals using transcranial color-coded real-time sonography. J Neuroimaging 1995;5:199-205.

12 Baumgartner RW, Arnold M, Gönner F, et al. Contrast-enhanced transcranial color-coded duplex sonography in ischemic cerebrovascular disease. Stroke 1997;28:2473-8

13 De Bray JM, Glatt B. Quantification of atheromatous stenosis in the extracranial internal carotid artery. Cerebrovasc Dis 1995:5:414-26.

14 Von Reutern GM, Von Büdingen HJ. Ultrasound diagnosis of cerebrovascular disease. Stuttgart: Thieme, 1993:208-48.

15 Lammie A, Sandercock P, Dennis M. Recently occluded intracranial and extracranial carotid arteries. Relevance of the unstable atherosclerotic plaque. Stroke 1999;30:1319-25.

16 Droste D, Junker K, Hansberg T, et al. Circulating microemboli in 33 patients with intracranial arterial stenosis. Cerebrovasc Dis 2002;13:26-30.

17 Nabavi D, Georgiadis D, Mumme T, et al. Detection of microembolic signals in patients with middle cerebral artery stenosis by means of a bigate probe. Stroke 1996;27:1347-9.

18 Sliwka U, Klötzsch C, Popescu O, et al. Do chronic middle cerebral artery stenoses represent an embolic focus? Stroke 1997;28:1324-7.

19 The Extracranial-Intracranial Bypass Study Group. Failure of extracranialintracranial bypass to reduce risk of ischemic stroke. Results of an international randomized trial. N Engl J Med 1985;313:1191-2000.

20 Bogousslavsky J, Barnett HJM, Fox J, et al. Atherosclerotic disease of the middle cerebral artery. Stroke 1986;17:1112-20.

21 Arenillas J, Molina C, Montaner J, et al. Progression and clinical recurrence of symptomatic cerebral artery stenosis. A long-term follow-up transcranial Doppler ultrasound study. Stroke 2001;32:2898-904.

22 Gorelick P, Caplan L, Hier D, et al. Racial differences in the distribution of anterior circulation occlusive disease. Neurology 1984;34:54-9. 